\title{
Ungual Tuberculosis: A Unique Clinical Case
}

\author{
Ana Cristina da Silva Sousa ${ }^{a}$ Manuel Sousa ${ }^{b}$ David Tente $^{c}$ Nuno Menezes ${ }^{a}$ \\ Armando Baptista $^{a}$ Rita Guedes ${ }^{a}$ \\ a'Dermatology Department, Centro Hospitalar Vila Nova de Gaia/Espinho, Vila Nova de Gaia, Portugal; \\ bPneumology Department of Vila Nova de Gaia, Vila Nova de Gaia, Portugal; 'Pathology Department, \\ Centro Hospitalar Vila Nova de Gaia/Espinho, Vila Nova de Gaia, Portugal
}

\section{Established Facts}

- Cutaneous manifestations of tuberculosis (TB) are rare.

- Involvement of the nail apparatus is extremely rare.

- The best treatment has yet to be established.

\section{Novel Insights}

- TB can be involved in onychodystrophy of the nail apparatus.

- TB infection should be considered as a diagnosis in cases of painless paronychia.

- Treatment with rifampicin, isoniazid, pyrazinamide, and ethambutol for 9 months leads to complete resolution.

\section{Keywords}

Cutaneous tuberculosis · Nail disease · Onychodystrophy · Paronychia

\section{Abstract \\ Cutaneous manifestations of tuberculosis (TB) are rare, par- ticularly from an exogenous source. Involvement of the nail apparatus is extremely rare and has only previously been re- ported as a secondary involvement. We report the case of a 76-year-old female patient referred to our department with onychodystrophy with purulent drainage of the first left fin- ger, which had developed during the preceding year. She}

had no previous traumatic history and had received treatment with multiple cycles of oral antibiotics and antimycotics, with no clinical improvement. Physical examination showed paronychia and onychodystrophy of the entire nail plate. Biopsy evaluation revealed epithelioid granulomas with central foci of necrosis, and laboratory cultures were positive for Mycobacterium tuberculosis complex. Chest computed tomography excluded primary pulmonary TB. X-ray of the left hand revealed the presence of dactylitis on the distal phalanx. Based on these findings, the patient was treated with rifampicin, isoniazid, pyrazinamide, and ethambutol for 2 months and with rifampicin and isoniazid for 7 months, resulting in complete resolution of the lesions. Cutaneous TB

\section{KARGER}

(C) 2019 S. Karger AG, Basel

E-Mail karger@karger.com

www.karger.com/sad
Ana Cristina da Silva Sousa

Dermatology Department, Centro Hospitalar Vila Nova de Gaia/Espinho Rua Conceição Fernandes

PT-4434-502 Vila Nova de Gaia (Portugal)

E-Mail a.cristina.silvasousa@gmail.com 


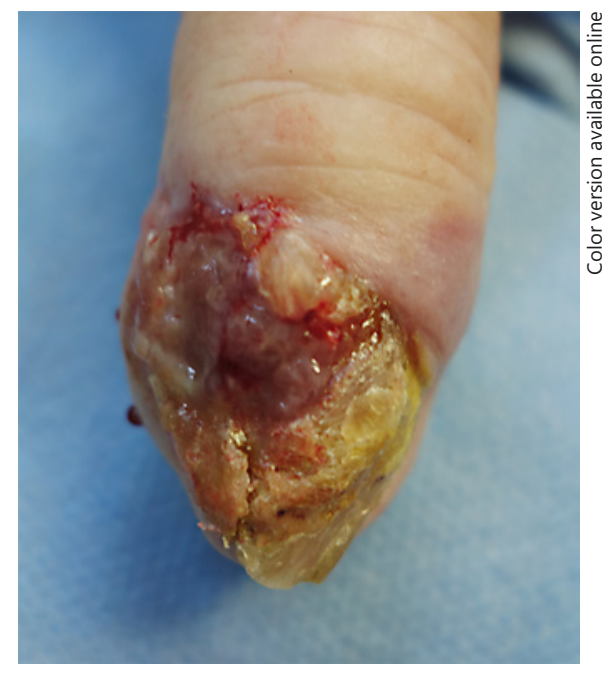

Fig. 1. Onychodystrophy of the entire nail plate with exuberant granulation tissue extending from the proximal nail fold to the nail plate, plus associated paronychia.

is a diagnostic challenge, particularly in rare cases such as involvement of the nail apparatus. It should be considered as a diagnostic hypothesis in cases of painless paronychia with refractory purulent drainage and associated onychodystrophy.

(c) 2019 S. Karger AG, Basel

\section{Introduction}

Although the incidence of tuberculosis (TB) in Portugal is declining, it remains an active public health problem affecting not only immunocompromised but also immunocompetent individuals. Cutaneous TB is a rare and poorly defined entity, accounting for 1-1.5\% of all extrapulmonary manifestations. Cutaneous TB can be classified into three groups: inoculation of TB from an exogenous source (also known as tuberculous chancre in immunosuppressed patients and TB verrucosa cutis in immunocompetent hosts); TB from an endogenous source, previously described as scrofuloderma, where the primary inoculation is commonly in the bone or lymph nodes; and haematogenous $\mathrm{TB}$, which accounts for the majority of cutaneous TB cases [1].

Tuberculous dactylitis is the involvement of the small tubular bones of the hands and feet, which is a rare presentation of skeletal TB and is most commonly described in children younger than 6 years of age [2]. The typical clinical presentation is a painless swelling of the digit that has been present for some months [3]. Involvement of the

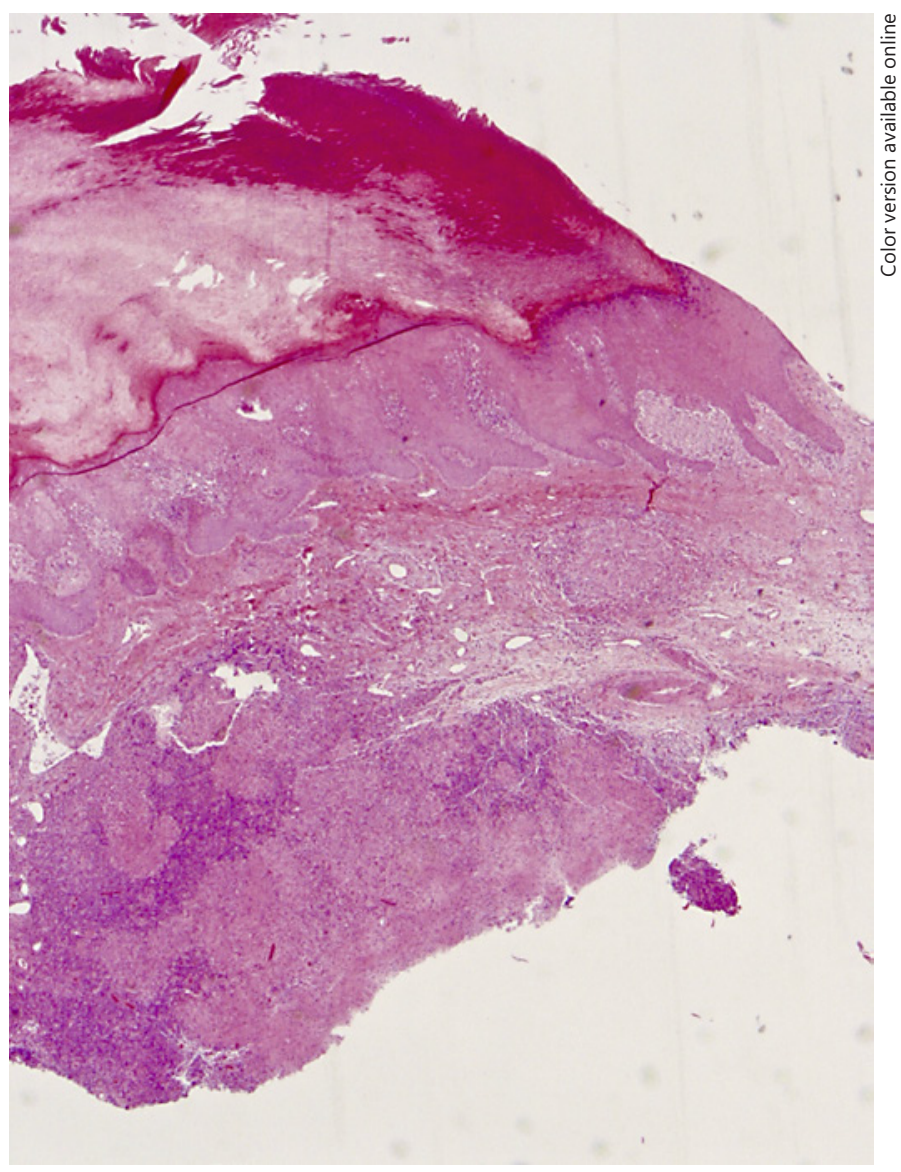

Fig. 2. Epithelioid granulomas with foci of necrosis on the dermis. $\mathrm{H} \& \mathrm{E}, 40 \times$.

nail apparatus secondary to tuberculous dactylitis has been reported only once previously in a paediatric patient [4].

\section{Case Report}

We present the case of a 76-year-old female patient, referred to our department with onychodystrophy with purulent drainage of the first finger of the left hand, which had developed during the course of the previous year. The patient had no knowledge of having been in direct contact with someone with TB or a history of previous $\mathrm{TB}$, and experienced no trauma, fever, night sweats, cough, or weight loss in the previous year. She had received treatment with multiple cycles of oral antibiotics and antimycotics, which had resulted in no clinical improvement. Physical examination revealed swelling and erythema of the first finger of the left hand with mild tenderness, onychodystrophy of the whole nail plate with exuberant granulation tissue extending from the proximal nail fold to the nail plate and associated paronychia (Fig. 1). The diagnoses considered were a nail tumour (squamous cell car- 


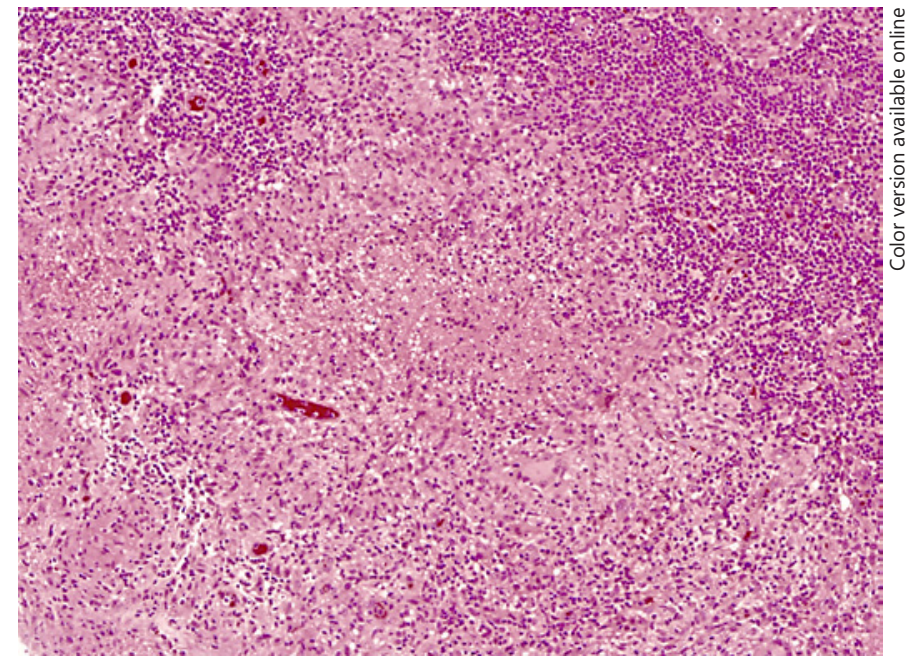

Fig. 3. Large epithelioid granuloma with central suppurative necrosis. H\&E, 100×.

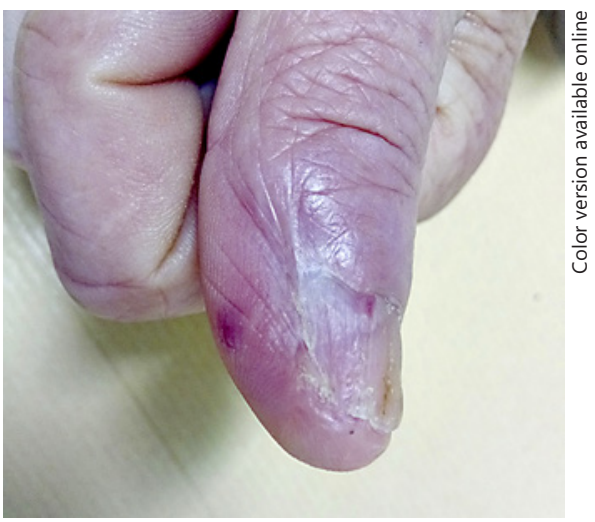

Fig. 4. Following 9 months of treatment with antituberculostatic therapy.

cinoma or amelanotic melanoma) or a nail infection (bacterial, fungal, or mycobacterial). A lateral longitudinal nail biopsy was performed, which revealed epithelioid granulomas with foci of necrosis (Fig. 2, 3). Ziehl-Neelsen (ZN) stain was negative.

Laboratory cultures were positive for Mycobacterium tuberculosis complex, but chest X-ray and chest computed tomography excluded primary pulmonary TB. The blood work was unremarkable, with no leuocytose or elevation of C-reactive protein. Serology for human immunodeficiency virus was nonreactive. X-ray of the left hand showed erosion of the cortical bone, osteopenia, and irregularities of the bone marrow of the first finger of the left hand, primarily in the distal phalanx. Based on these findings, the patient was treated with rifampicin, isoniazid, pyrazinamide, and ethambutol for 2 months and with rifampicin and isoniazid for 7 months, resulting in complete resolution of the lesions (Fig. 4).

\section{Discussion}

Primary cutaneous TB results from direct inoculation of $M$. tuberculosis through the skin barrier [5]. The first case of cutaneous TB was described in 1826 by Théophile Laennec, when he described his own "prosector's warts." [6]. The lesions typically begin with a painful, small erythematous papule that progresses into a warty lesion, which is typically asymptomatic, or an ulcer that can be very small or up to $5 \mathrm{~cm}$ in diameter [7].

Painless paronychia due to primary TB infection has been reported in healthy subjects $[8,9]$. The nail fold is particularly vulnerable to frequently minor trauma, potentially leading to direct inoculation of $M$. tuberculosis in these patients. Secondary cutaneous TB results from a subcutaneous focus, typically a lymph node or bone, that progresses to the adjacent skin. The lesions start as a subcutaneous nodule with discharge and subsequently progress to a cutaneous abscess [5]. These clinical manifestations are more common in children and patients with a systemic TB infection, particularly pulmonary $\mathrm{TB}$, which has been described in $35 \%$ of cases [10, 11].

Histologically, typical granulomatous tubercles with epithelioid cells, Langerhans giant cells, and mononuclear infiltrate are the most common features, although isolation of the microorganism by culture testing is imperative for a definitive diagnosis [12]. The treatment recommended by the WHO consists of two phases; the first is an intensive 8-week course of isoniazid, rifampicin, ethambutol, and pyrazinamide, followed by a 16 -week maintenance phase with isoniazid and rifampicin [1]. To date, only one case of tuberculous dactylitis with a secondary paronychia has been reported in the literature [4]. Although the case is similar to that presented here, it is located in the hallux and is reported in a paediatric patient, which is the most common age of presentation. However, the case reported here is of particular interest as it demonstrates involvement of both the bone and nail apparatus in an immunocompetent patient with no systemic TB infection. Although it is not possible to determine with any certainty whether the nail involvement is primary or secondary TB, the hands are a common site of trauma and therefore direct inoculation. 


\section{Conclusions}

Cutaneous TB presents a significant diagnostic challenge, particularly if the nail apparatus is involved, given the rarity of this condition. It should, however, be considered as a diagnostic hypothesis in cases of painless paronychia with refractory purulent drainage and associated onychodystrophy.

\section{Acknowledgements}

Bioedit Ltd. participated in the technical editing of the manuscript.

\section{Statement of Ethics}

The authors declare that the patient gave written informed consent to publish this case report (including publication of images).

\section{Disclosure Statement}

The authors have no conflicts of interest to declare.

\section{Funding Sources}

The authors declare that no funding sources supported the work.

\section{Author Contributions}

All authors participated in the collection of clinical, analytical, and imaging data, which was indispensable for arriving at a diagnosis and played an important role in the clinical orientation of the patient. Cristina Sousa wrote the manuscript. All authors have read and approved the manuscript.

\section{References}

1 van Zyl L, du Plessis J, Viljoen J. Cutaneous tuberculosis overview and current treatment regimens. Tuberculosis (Edinb). 2015 Dec; 95(6):629-38.

2 Tuli SM. Tuberculosis of the skeletal system (bones, joints, spine and bursal sheaths). 2nd ed. New Delhi: Jaypee Brothers Medical Publishers Ltd; 1991. p. 159-161.

3 Hardy JB, Hartmann JR. Tuberculous dactylitis in childhood; a prognosis. J Pediatr. 1947 Feb;30(2):146-56.

4 Khanna D, Chakravarty P, Agarwal A, Gupta $\mathrm{R}$. Tuberculous dactylitis presenting as paronychia with pseudopterygium and nail dystrophy. Pediatr Dermatol. 2013 Nov-Dec; 30(6):e172-6
5 Hill MK, Sanders CV. Cutaneous Tuberculosis. Microbiol Spectr. 2017 Jan;5(1). https:// doi.org/10.1128/microbiolspec. TNMI70010-2016.

6 Rullán J, Seijo-Montes RE, Vaillant A, Sánchez NP. Cutaneous manifestations of pulmonary disease. In: Sánchez NP, editor. Atlas of dermatology in internal medicine. 14th ed. San Juan, Puerto Rico: Springer; 2012. p. 1730. https://doi.org/10.1007/978-1-46140688-4_2.

7 Ramarao S, Greene JN, Casanas BC, Carrington ML, Rice J, Kass J. Cutaneous manifestations of tuberculosis. Infect Dis Clin Pract. 2012 Nov;20(6):376-83.

8 Goette DK, Jacobson KW, Doty RD. Primary inoculation tuberculosis of the skin. Prosector's paronychia. Arch Dermatol. 1978 Apr; 114(4):567-9.
9 O'Donnell TF Jr, Jurgenson PF, Weyerich NF. An occupational hazard-tuberculous paronychia. Report of a case. Arch Surg. 1971 Dec; 103(6):757-8.

10 Kim GW, Park JH, Kim HS, Kim SH, Ko HC Kim BS, et al. Delayed diagnosis of scrofuloderma misdiagnosed as a bacterial abscess. Ann Dermatol. 2012 Feb;24(1):70-3.

11 Verma S, Thakur BK, Gupta A. Multifocal childhood cutaneous tuberculosis: report of two interesting cases from Sikkim, India. Pediatr Dermatol. 2013 May-Jun;30(3):e1-4.

12 Semaan R, Traboulsi R, Kanj S. Primary Mycobacterium tuberculosis complex cutaneous infection: report of two cases and literature review. Int J Infect Dis. 2008 Sep;12(5):472-7. 